\title{
Emergence of Klebsiella pneumoniae ST307 Co- Producing CTX-M with SHV and KPC from Paediatric Patients at Shenzhen Children's Hospital, China
}

\author{
Sandip Patil (iD ${ }^{1-3}$ \\ Hongyu Chen ${ }^{4}$ \\ Chunna Guo 4 \\ Xiaoli Zhang ${ }^{3}$ \\ Pei-Gen Ren' \\ Ngiambudulu M Francisco iD ${ }^{5}$ \\ Feiqiu Wen ${ }^{2,3}$ \\ 'Shenzhen Institute of Advanced \\ Technology, Shenzhen University Town, \\ Shenzhen, Guangdong Province, People's \\ Republic of China; ${ }^{2}$ Department of \\ Haematology and Oncology, Shenzhen \\ Children's Hospital, Shenzhen, \\ Guangdong Province, People's Republic \\ of China; ${ }^{3}$ Paediatric Research Institute, \\ Shenzhen Children's Hospital, Shenzhen, \\ Guangdong Province, People's Republic \\ of China; ${ }^{4}$ Department of Laboratory \\ Medicine, Shenzhen Children's Hospital, \\ Shenzhen, Guangdong Province, People's \\ Republic of China; ${ }^{5}$ Grupo de \\ Investigação Microbiana e Imunológica, \\ Instituto Nacional de Investigação em \\ Saúde (National Institute for Health \\ Research), Luanda, Angola
}

\begin{abstract}
Aim: We investigated the clonal diversity of carbapenemase-producing Klebsiella pneumoniae isolates from the Shenzhen Children's Hospital, China, and drew conclusions on the clinical and public health impact of these isolates as multidrug-resistant.

Methods: From January 2014 to December 2018, a total number of 36 unique carbapenemase-producing clinical isolates of Klebsiella pneumoniae were collected out of 900 clinical isolates in paediatric patients from the Shenzhen Children's Hospital, China. After carbapenemase production confirmation, antimicrobial susceptibility, resistance determinants and phylogenetic relationship were determined.

Results: The isolates showed resistance to ceftazidime, ertapenem, ampicillin, cefazolin, ceftriaxone, cefotetan, ticarcillin, cefaclor, cefpodoxime, azlocillin, cefcapene, mezlocillin and ampicillin-sulbactam. Of the 36 Klebsiella pneumoniae carbapenemase genes coding isolates, bla $a_{\mathrm{NDM}}$ was the mostly detected $50 \%(\mathrm{n}=18)$ followed by $b l a_{\mathrm{KPC}}$ and $b l a_{\mathrm{IMP}} 19 \%(\mathrm{n}=7)$, bla $a_{\mathrm{VIM}} 17 \%(\mathrm{n}=6)$, $b l a_{\text {OXA-48-like }} 8 \%(\mathrm{n}=3)$ and $b l a_{\mathrm{SME}} 5 \%(\mathrm{n}=2)$, whereas extended-spectrum $\beta$-lactamase $\left(b l a_{\mathrm{SHV}}\right)$ was predominantly detected $92 \%(\mathrm{n}=33)$ followed by $b l a_{\text {CTX-M }} 53 \%(\mathrm{n}=19)$ and bla $_{\mathrm{CMY}} 28 \%$ $(n=10)$. Pulsed-field gel electrophoresis typing showed eight different patterns, and twenty-five distinct sequences types were observed with ST307 being predominantly identified $11 \%(n=4)$, followed by ST2407 8\% (n=3). Plasmid replicon typing results indicated that IncFIS, IncHI2, IncFIC and IncFIA plasmids carry $b l a_{\mathrm{CTX}-\mathrm{M}}, b l a_{\mathrm{SHV}}$ and $b l a_{\mathrm{NDM}}$ genes.

Conclusion: This study reports on the occurrence and spread of carbapenemase and extended-spectrum $\beta$-lactamase encoding genes co-existence in sporadic Klebsiella pneumoniae ST307 in paediatric patients from the Shenzhen Children's Hospital, China.
\end{abstract}

Keywords: Klebsiella pneumoniae, carbapenemase, ESBLs, antimicrobial susceptibility, molecular characterization

\section{Introduction}

The rise and spread of antimicrobial resistance bacteria are universal symbolic challenges for healthcare due to the restricted treatment choices. ${ }^{1}$ In the past year, carbapenemresistant Klebsiella pneumoniae (CRKP) infections have become a growing of global public health concern, particularly in paediatric patients, due to high morbidity and mortality. ${ }^{2}$ Carbapenemase enzymes encoded by alleles of the bla $a_{\mathrm{KPC}}$ gene, depict one of the five substantial carbapenemase families, others being the VIM, IMP and New Delhi metallo- $\beta$-lactamase (M $\beta$ L) (NDM), also, the OXA-48-like oxacillinases have
Correspondence: Feiqiu Wen

Department of Haematology and

Oncology, Shenzhen Children's Hospital,

Shenzhen, Guangdong Province, People's

Republic of China

Tel $+86 \quad 18938690333$

$\mathrm{Fax}+86-75583009888$

Email fwen62@163.com 
been reported worldwide. ${ }^{3,4}$ The commonness of each carbapenemase differs geographically, and its resistance profiles vary. $^{5,6}$ Several Klebsiella pneumoniae carbapenemase (KPC) outbreaks were reported in Brazil, Argentina, Poland, Germany, France, Spain and China (Jiangsu), ${ }^{7}$ whereas NDM producing Klebsiella pneumoniae is highly prevalent in Canada, Greece, Belgium, Sweden, Norway, India, Pakistan, Bangladesh, Korea and China. ${ }^{8}$ Thus, OXA-48-like oxacillinases producing strains are widely spread in Turkey, Morocco and France, and the VIM is highly spread in Greece. ${ }^{9}$ In China, despite the considerable dissemination of KPC-producing strains, NDM-producing $K$. pneumoniae are the type mainly found in children, and are seldom reported in adults. ${ }^{10}$ The CHINET data report of 2005-2014 reported that the detection rate of CRKP isolates in paediatric patients has increased from $5.3 \%$ to $15.9 \%{ }^{11}$ Recently, K. pneumoniae isolates producing OXA-232, OXA-163 and OXA-405 carbapenemase were reported in Children's Hospitals globally including China. ${ }^{12,13}$ Carbapenem antibiotics are the foremost alternative to treat conditions caused by multidrug-resistant (MDR) bacteria due to the side effects of other drugs, such as fluoroquinolones and aminoglycosides. ${ }^{14}$ This has led to an exponential increase in carbapenem resistance among pathogenic bacteria. Despite the fact that CRKP has spread globally and has serious clinical outcomes in pediatric patients, there are insufficient data available on the susceptibility and genetic platform of carbapenemase production of $K$. pneumoniae in China. CRKP has been significantly increased in China, of particular concerns is the emerging multiple carbapenemase encoding genes in single isolates or co-existence extended spectrum $\beta$-lactamase (ESBLs), and the recently discovered colistin resistance plasmid-born $m c r-1$; which results in highly drug-resistant strains (Pan-drug-resistant strains) that are probably untreatable. ${ }^{15}$ Carbapenemase encoding genes detection significantly aids in targeted drug use, and helps to prevent further dissemination. Thus, this study aimed to investigate the resistance gene profile, molecular epidemiology, and clinical characteristics of CPKP isolates obtained from paediatric patients in the Shenzhen's Children Hospital in China.

\section{Materials and Methods}

\section{Clinical Sample, Bacterial Isolation and Identification}

Thirty-six unique (one isolate from one patient) clinical isolates of K. pneumoniae were collected from January 2014 to December 2018 from Shenzhen Children's Hospital, Shenzhen, China. Among the 36 CRKP isolates, 61\% $(n=22)$ were from male and $39 \%(n=14)$ were from female patients with ages ranging from one month to twelve years. The clinical isolation type of specimens was as follows: urine $44 \%(n=16)$, sputum 25\% $(n=9)$, blood $12 \%(n=4)$ and catheter-associated secretion (CAS) $6 \%(n=2)$, while the cerebral-spinal fluid (CSF), throat swab (TS), pus, and abdominal fluid or secretion each had $13 \%(n=1)$. This study was conducted in accordance with the declaration of Helsinki.

All isolates were primarily identified by API20E (Biomerieux, Ref. No.27530/275660) automated system and further confirmed by using 16s RNA gene sequence primers.

\section{Phenotypic Detection of CRKP}

Carbapenemase production was confirmed by using a newly developed carbapenem inactivation method (CIM) as delineated in the year $2015 .{ }^{16}$ To carry out the CIM, an antibiotic susceptibility-testing disc of $10-\mu \mathrm{g}$ meropenem (MEM) was incubated for $2 \mathrm{hrs}$ in an aqueous suspension of a $K$. pneumoniae. The disc was removed from the suspension and placed onto a Mueller-Hinton agar (MHA) plate, seeded with an ATCC25922 indicator organism, followed by overnight incubation. The zone of inhibition was measured to determine whether the MEM had been hydrolyzed (growth of the indicator organism close to the disc), or still active (a large zone of inhibition around the disc). The positive control strain was selected from the collected isolates characterized from our laboratory.

\section{Phenotypic Detection of ESBLs Production}

The ESBLs production was determined by using VITEK@2 compact system (Biomerieux, Ref. No. 27530/275660). We used a control strain that was characterized from our laboratory, while ATCC25922 was used as a negative control strain. The ESBLs production result was analyzed according to the Clinical and Laboratory Standards Institute (CLSI) guideline. ${ }^{17}$

\section{Antimicrobial Susceptibility}

Antimicrobial susceptibility was performed by using VITEK@2 compact system (Biomerieux, Ref. No. 27530/ 275660) for 25 antimicrobial agents, namely ampicillin/sulbactam, piperacillin, ertapenem, amikacin, levofloxacin, nitrofurantoin, ampicillin, cefazolin, ceftazidime, ceftriaxone, cefepime, imipenem, cefotetan, tobramycin, gentamicin, ticarcillin, cefaclor, cefpodoxime, azlocillin, cefcapene, 
mezlocillin, trimethoprim, aztreonam, and ciprofloxacin. Colistin susceptibility was performed by the disc diffusion method. To further ascertain the antimicrobial susceptibility, we used CIM disc to detect carbapenemase enzyme, where the disc was removed from the suspension and placed onto a Mueller-Hinton agar (MHA) plate, seeded with an ATCC25922 indicator organism, followed by overnight incubation. The results were construed according to the Clinical and Laboratory Standards Institute (CLSI) guideline (CSLI, 2019) ${ }^{16}$ and EUCAST 2016. ${ }^{18}$

\section{Detection of Carbapenemase Encoding Genes}

The standard PCR was used to detect the presence of carbapenemase encoding genes. Class-A includes $b l a_{\mathrm{IMI}}, b l a_{\mathrm{GES}}$, $b l a_{\mathrm{SME}}$ and $b l a_{\mathrm{KPC}}$, while Class-B consists of bla $a_{\mathrm{IMP}}, b l a_{\mathrm{VIM}}$, $b l a_{\mathrm{GIM}}, b l a_{\mathrm{SIM}}$ and $b l a_{\mathrm{NDM}}$ and Class-D made up of $b l a_{\mathrm{OXA}-48}$ like using specific primers as previously described. ${ }^{19-21}$ In addition, PCR assay was carried out for other $\beta$-lactamase encoding genes: bla $a_{\mathrm{CTX}-\mathrm{M} \text {-(variant), }}$ la $_{\mathrm{SHV}}, b l a_{\mathrm{CMY}}, b l a_{\mathrm{TEM}}$ and $b l a_{\mathrm{VEB}}$ by using specific primers as described earlier. ${ }^{22}$ Control strain, which was selected from the characterized strain collection of our laboratory. The private company (Sangon Biotech-Shanghai, China) sequenced the purified PCR products. DNA sequences were analyzed using the following URLs https://blast.ncbi.nlm.nih.gov/Blast.cgi, http:// www.bldb.eu:4567/ and https://bigsdbpasteur.fr/klebsiella/ klebsiella.htmL.

\section{PFGE and Multi-Locus Sequence Typing (MLST)}

We performed PFGE to check whether there is a presence of any clonal transmission within the Hospital. Furthermore, we used the MLST to assess the genetic relatedness of the identified isolates.

\section{DNA Extraction Details}

Post-extraction DNA was digested with 45U Xbal (Takara Biotech) for 2 hours at $37^{\circ} \mathrm{C}$. We used CHEFDRIII apparatus (Bio-Rad Laboratories, Hercules, CA, USA) to perform PFGE for $K$. pneumoniae isolates as previously described. $^{23}$ PCR assay was performed to amplify internal portions of the seven housekeeping genes of $K$. pneumoniae (gapA, infB, mdh, pgi, phoE, rpoB, and tonB) with specific primers. ${ }^{24}$ Amplified products were sequenced from private enterprise (Sangon Biotech-Shanghai, China http://www.pas
teur.fr/recherche/genopole/PF8/mlst/Kpneumoniae) and were referred to assign sequence types (STs).

\section{Plasmid Transferability}

Streptomycin-resistant $E$. coli $\mathrm{C}_{600}$ was used as the recipient strain in conjugation experiments to analyze the horizontal genes transformation of plasmid-borne $\beta$-lactamase encoding genes in CRKP isolates, using liquid mating assay as previously described. ${ }^{14}$ Transconjugants were selected using Luria Bertani agar containing streptomycin $2000(\mu \mathrm{g} / \mathrm{mL})$, ampicillin $(100 \mu \mathrm{g} / \mathrm{mL})$ and cefotaxime (32 $\mu \mathrm{g} / \mathrm{mL})$. Therefore, we further tested them using PCR for $\beta$-lactamase encoding genes after performing a phenotypic combination disc test.

\section{PCR-Based Replicon Typing}

PCR-based replicon typing was performed for both plasmids from parental and transconjugant isolates. The Inc (incompatibility) groups were determined by using a specific primer as previously described by Carattoli et al $2005 .^{25}$

\section{Results}

\section{Antimicrobial Resistance Profile of K. pneumoniae}

All the 36 isolates were identified as $K$. pneumoniae using API20E (Biomerieux, Ref. No. 27530/275660) automated system and were further confirmed using 16s RNA gene sequencing. Antimicrobial susceptibility tests were found in all the 36 CRKP isolates $(100 \%)$ and were all resistant to ceftazidime, ertapenem, ampicillin, cefazolin, ceftriaxone, cefotetan, ticarcillin, cefaclor, cefpodoxime, azlocillin, cefcapene, mezlocillin and ampicillin-sulbactam. However, aztreonam showed 89\% $(n=32)$, piperacillin $86 \%(n=31)$, cefepime $83 \%(n=30)$, imipenem $80 \%(n=29)$, nitrofurantoin $47 \%(n=17)$, trimethoprim $44 \%(n=16)$, ciprofloxacin, gentamicin, levofloxacin 30\% $(n=11)$, tobramycin $25 \%(n=9)$, amikacin $11 \%(n=4)$. All isolates were susceptible to colistin (Figure 1), and the $36 \mathrm{CRKP}$ isolates showed a multi-drug resistant phenotype, hence designated as "superbugs".

\section{Molecular Analysis of Drug Resistance Genes}

All the 36 CRKP isolates were carrying carbapenemase encoding genes, with the most common being $b l a_{\mathrm{NDM}}$ $50 \%(\mathrm{n}=18)$ which include $b l a_{\mathrm{NDM}-1}(\mathrm{n}=13), b l a_{\mathrm{NDM}-6}$ $(\mathrm{n}=3)$ and bla $_{\mathrm{NDM}-5}(\mathrm{n}=2)$; followed by bla $_{\mathrm{IMP}} 19 \%$ 


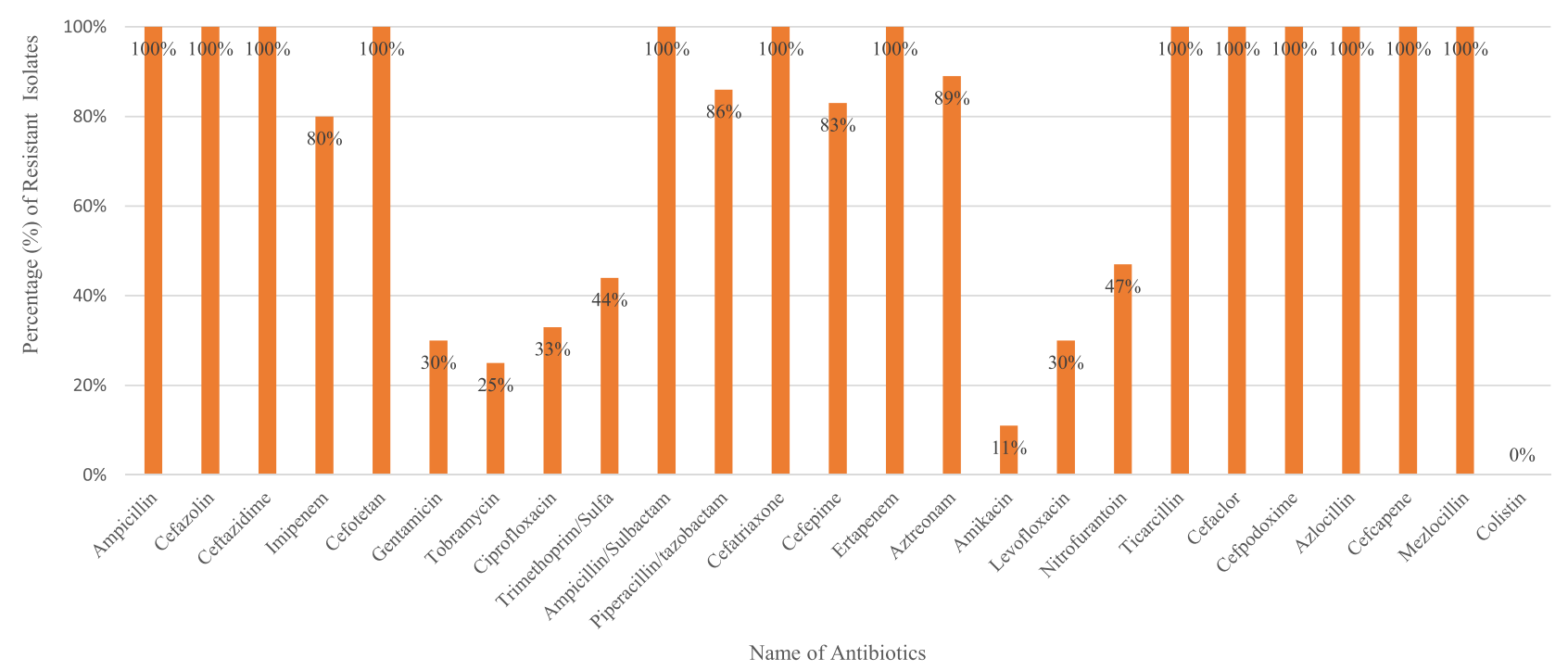

Figure I Antimicrobial resistance patterns of 25 commonly used antibiotics against 36 carbapenemase-producing Klebsiella pneumoniae isolates from paediatric clinical cases.

$(\mathrm{n}=7)$ including bla $_{\mathrm{IMP}-38}(\mathrm{n}=5)$ and bla $_{\mathrm{IMP}-4}(\mathrm{n}=2), \quad(\mathrm{n}=15)$, bla $_{\mathrm{SHV}-1}(\mathrm{n}=13)$, bla $_{\mathrm{SHV}-27}(\mathrm{n}=3), \quad b l a_{\mathrm{SHV}-26}$ bla $_{\mathrm{KPC}-2} 19 \%(\mathrm{n}=7)$, bla $_{\mathrm{VIM}-2} 17 \%(\mathrm{n}=6)$, bla $_{\mathrm{OXA}-48}$ and bla $_{\mathrm{SHV}-33}(\mathrm{n}=1)$, bla $_{\mathrm{CTX}-\mathrm{M}}(\mathrm{n}=19), \quad b l a_{\mathrm{CTX}-\mathrm{M}-3}$ and bla $_{\mathrm{SME}-1} \quad 8 \% \quad(\mathrm{n}=3)$ each. Additionally, co- $(\mathrm{n}=11), b l a_{\mathrm{CTX}-\mathrm{M}-15}(\mathrm{n}=5)$, bla $_{\mathrm{CTX}-\mathrm{M}-14,}, 26,40, \quad(\mathrm{n}=1$ existences of other $\beta$-lactamases encoding genes were each), bla $a_{\mathrm{CMY}-2} 28 \%(\mathrm{n}=10)$ (Figure 2). The $b l a_{\mathrm{IMI}}$, detected in $b l a_{\mathrm{SHV}} 92 \%(\mathrm{n}=33)$, contains $b l a_{\mathrm{SHV}-11} \quad b l a_{\mathrm{GES}}, b l a_{\mathrm{GIM}}, b l a_{\mathrm{SIM}}$ carbapenemase encoding genes

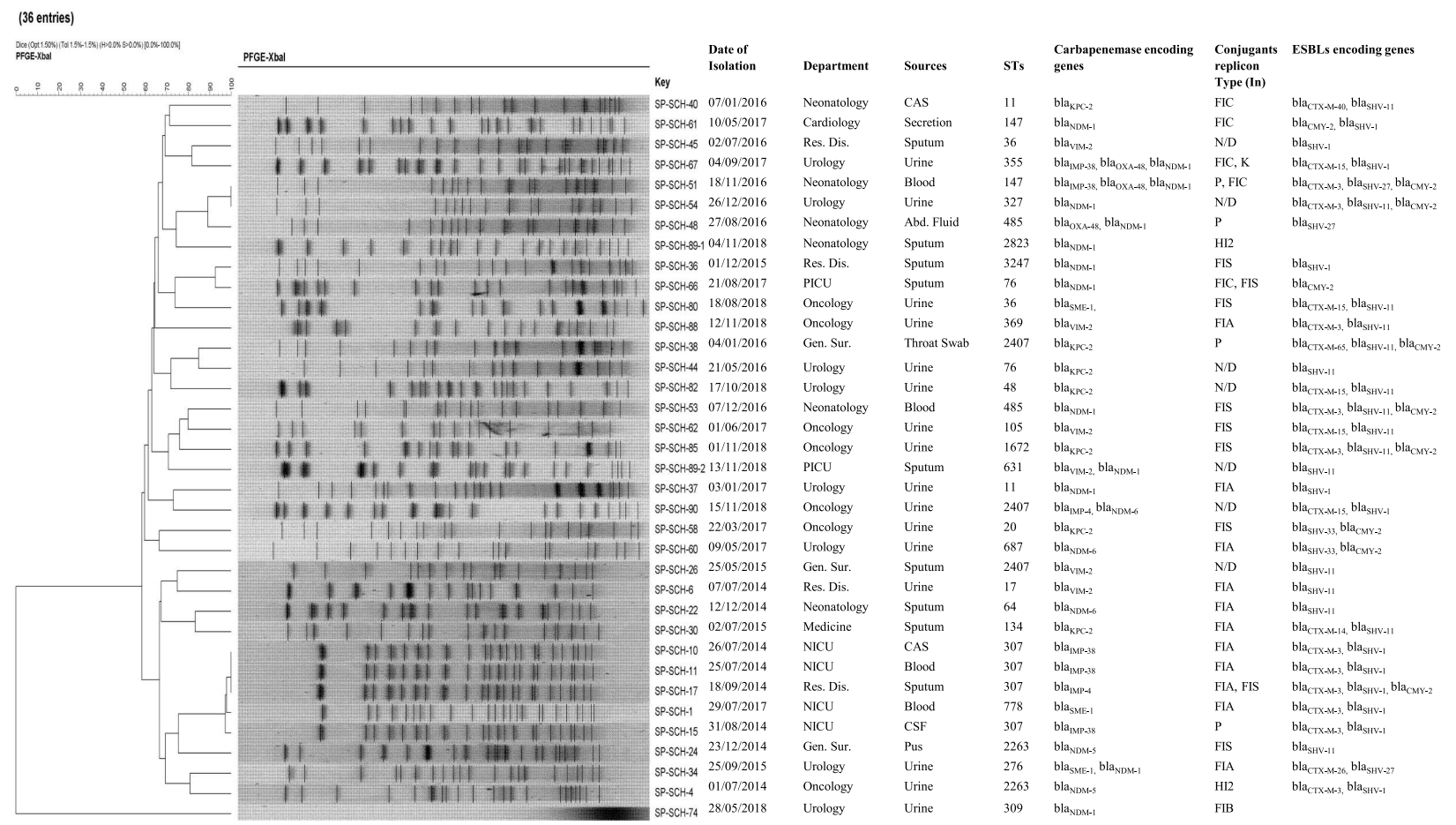

Figure 2 Dendrogram of the 36-PFGE-X-bal identified CRKP isolates collected from paediatric patients showing their genetic relatedness by date of isolation, department of isolation, sources of specimen, sequences types, replicon type, resistant determinants and clonal relatedness. 
and ESBLs encoding genes bla $a_{\mathrm{TEM}}, b l a_{\mathrm{VEB}}$ were not observed in this study. It is interesting that approximately $53 \%(n=19)$ of CRKP strains co-harbouring ESBLs encoding genes $b l a_{\mathrm{CTX}-\mathrm{M} \text {-variant }}$ were found in $b l a_{\mathrm{SHV}-\mathrm{variant}}$, with this, at least $50 \%$ of strains were carrying bla $a_{\mathrm{NDM} \text {-variant }}$.

\section{Multi-Locus Sequences Typing and PFGE}

Extensive diversity of MLST was recorded from CRKP isolates. In 25 different STs, we found that ST307 isolates $(11 \%)$ were recovered from the neonatal intensive care unit (NICU). ST2407 (8\%) was found to be highly prevalent and were collected from the general surgery department (GSD). In addition, we found that CRKP ST307 was dominant in NICU and in a reservoir of $b l a_{\mathrm{CTX}-\mathrm{M}-3}$ and $b l a_{\mathrm{SHV}-11}$. However, ST2407 was predominantly in GSD, which is a key transporter for bla $\mathrm{CTX}_{\mathrm{C}-\mathrm{M}-15}$, and $b l a_{\mathrm{SHV}-11}$ gene and ST2263 a key transporter for bla $a_{\mathrm{NDM}-5}$. Nevertheless, blaKPC-2 was reported in diverse STs (Figure 2). Our particular concern is that the CRKP co-harbouring ESBLs encoding genes $b l a_{\mathrm{CTX}-\mathrm{M} \text {-variant }}$ and $b l a_{\mathrm{SHV} \text {-variant }}$ were reported in seventeen different STs in this hospital. This observation suggests that CRKP strains carrying ESBLs encoding genes might have spread to the Shenzhen region and may be widespread in Southern China. These 36 CRKP isolates were allocated to eight distinct PFGE clusters, sharing $\geq 80 \%$ of similar bands. The PFGE results showed that the clonal transmission was often observed within different departments of the Hospital (Figure 2).

\section{Plasmid Profiling}

In total, 18 out of 36 successful transconjugants were selected using Luria Bertani agar. PCR-based replicon typing assay results showed that Inc plasmids groups lead was IncFIA 55\% $(\mathrm{n}=10)$, followed by IncFIS $50 \%$ $(n=9)$, IncFIC 27\% $(n=5)$, IncP 22\% $(n=4)$, IncHl2 11\% $(\mathrm{n}=2)$, while IncK and IncFIB showed $6 \% \quad(\mathrm{n}=1)$ (Figure 2).

\section{Discussion}

CRKP is a serious threat in paediatric patients, particularly newborns, due to limited therapeutic options. ${ }^{26,27}$ This pathogen is now considered as a reservoir for virulent and resistant genes, due to the acquisition of $\beta$-lactamase (carbapenemase and ESBLs) and the recently reported colistin resistance $m c r-1$ encoding genes; which make it a serious threat to human and animal. $^{28-30}$ These data revealed for the first time that NDM carbapenemase is widely spread among CRKP, while CTX-M and SHV were the most prevalent $\beta$ lactamase in same isolates in Shenzhen's children Hospital, China. So far, there are no data available on molecular analysis of CRKP in paediatric clinical cases, particularly those caused by IMP or NDM co-production of CTX-M and SHV. Here, we first explored the genetic background of CRKP isolates obtained from the paediatric samples. Data showed that CRKP isolates were highly resistant to commonly used antibiotics, except for colistin. We also found that IMP-38 producing $K$. pneumoniae isolates are significantly sensitive to Imipenem, which is in agreement with studies reported in Australia, however further investigation is required. ${ }^{31}$ bla $a_{\mathrm{NDM}-1}$ was found to be the leading genotype of carbapenemase followed by $b l a_{\mathrm{KPC}-2}, b l a_{\mathrm{VIM}-2}, b l a_{\mathrm{IMP}-4}$, $b_{\text {NDM-6, }}, b l a_{\mathrm{SME}-1}, \quad b l a_{\mathrm{oxa}-48}, b_{\mathrm{IMP}-2}$, and $b l a_{\mathrm{NDM}-5}$. A previous study reported the presence of NDM-1 dominant carbapenemase in carbapenem-resistant $K$. pneumoniae over China, which is comparable to our results. $^{32}$ There were no cases with co-harbouring blaNDM-1 and bla $a_{\mathrm{IMP}-4}$ strain, which is in contrast with Liu et al's finding. ${ }^{33}$ Interestingly, nineteen CRKPs were found to contain bla $a_{\mathrm{CTX}-\mathrm{M}}$ encoding determinants. To the best of our knowledge, this is the first report on an MDR CRKP isolates co-harbouring $b l a_{\mathrm{NDM}}$, bla $a_{\mathrm{CTX}-\mathrm{M}}$ and $b l a_{\mathrm{SHV}}$ has flagged concerns about the spread of such superbugs in the Shenzhen region, China; but also, studies have reported similar results from other provinces of China, Chongqing and Shanghai. ${ }^{34,35}$ The MLST results revealed that $K$. pneumoniae ST307 and ST2407 were highly dominant in Shenzhen area, which encodes carbapenemase and ESBLs genes. Several countries such as Italy, Korea, USA, Mexico, and China have reported CRKP ST307 with ESBLs production. ${ }^{36-40}$ No study has yet reported that the isolate CRKP belonged to ST307 in the Southern region of China. The emergence of this isolate indicates the rapid spread of this isolate. Also, studies have reported the existence of CRKP belonged to ST2407. Data also report the presence of CRKP belonged to ST2407; to the best of our knowledge, no study has yet reported the presence of CRKP ST2407 isolates that exist with ESBLs in Southern China. Our finding of CRKP belonged to ST307 and ST2407 isolates, identify a substantial public health concern. 
Clonal dissemination within the hospital corresponded with a previous study by Tian et al 2018 in Shanghai Province, China. ${ }^{41}$ We did not analyze isolates from other hospitals to observe whether there was clonal transmission between the hospitals. Plasmid replicon typing and conjugation experiment results conferred that IncFIA, IncFIS, IncFIC, IncHI2, IncFIB, IncK and IncP replicons existed in the transconjugants and carbapenemase coding genes $\left(b l a_{\mathrm{KPC}-2}\right.$, and $\left.b l a_{\mathrm{NDM}-\mathrm{variants}}\right)$ co-transferred with ESBLs encoding genes ( $b l a_{\mathrm{CTX}-\mathrm{M} \text {-variant }}$ and $\left.b l a_{\mathrm{SHV} \text {-variant }}\right)$. No apparent relationship between replicon and sequence type was observed in the current isolates. Also, data of Figure 2 show the presence of some previously published isolates with co-production of carbapenemases, which requires further analysis as these isolates could be of clinical concern. Previously published study has done successful clone for KPC such as the clone group GC258, ${ }^{42}$ nevertheless from our $36 \mathrm{KPC}$ isolates reported in our study, none of them corresponded to the clone group GC258. And we did not find the dissemination of any particular clones at the hospital in Shenzhen, China. Our finding stresses out the importance of continuous monitoring to detect multi-drug resistant isolates to promote therapeutic strategies for infections in paediatric patients. Future studies may assess the presence of other resistance-related determinants by using WGS, such as the outer-membrane permeability and to augment sample size from different areas for the molecular study. It is also worth reporting here that although the methods used in our study are adequate, one of the potential limitations of this study is the lack of use of broth microdilution susceptibility testing method or the Clinical and Laboratory Standards Institute recommended disk elution method for colistin.

\section{Conclusion}

This study reports, for the first time, the emergence of CPKP ST307 co-producing CTX-M with SHV and KPC in paediatric patients from the Shenzhen's Children Hospital in China. Also, our results identify the occurrence of CRKP ST2407 belongs to co-producing CTX-M with SHV and KPC. The emergence of CPKP in our study highlights a substantial global concern, both within hospitals and the wider community. Continued appraisal of clinical experience and monitoring of the spread of CPKP will provide further important information on the emergence of CPKP ST307.

\section{Abbreviations}

Res. Dis., respiratory diseases; PICU, paediatric intensive care unit; Gen. Sur., general surgery; NICU, neonatal intensive care unit; CAS, catheter associated secretion; Abd., abdominal; CSF, cerebral spinal fluid; STs, sequences type; In, Incompatibility; N/D, not detected.

\section{Data Sharing Statement}

All data files mentioned in this manuscript have been submitted and are available.

\section{Ethics Approval and Consent to Participate}

The present study was approved by the Shenzhen Children's Hospital, Research Ethical Committee, reference number: 2018 (013).

\section{Consent for Publication}

The clinical isolate samples used in this research were part of the routine Hospital Laboratory procedure We do not use patients' name or personal information, so in the publication, no written consent was required. Verbal consent was freely taken from the patients' parents after being duly informed of its nature.

\section{Acknowledgments}

The authors would like to sincerely thank Prof. Ma Lian and Prof. Xiaowen Chen for their constant support. The abstract of this paper was presented at the 19th International Congress on Infectious Diseases as a poster presentation with interim findings. The poster's abstract was published in the International Journal of Infectious Diseases, 2020:101 (S1):57 [https://doi.org/10.1016/j.ijid.2020.09.181] .

\section{Author Contributions}

All authors made a significant contribution to the work reported, whether that is in the conception, study design, execution, acquisition of data, analysis and interpretation, or in all these areas; took part in drafting, revising or critically reviewing the article; gave final approval of the version to be published; have agreed on the journal to which the article has been submitted; and also agree to be accountable for all aspects of the work.

\section{Funding}

This study was funded by: 1. Shenzhen Industry and Information Committee "Innovation Chain and Industry 
Chain" integration special support plan project (Grant No: 20180225103240819), and 2. Science and Technology Project from the Science Technology and Innovation Committee of Shenzhen Municipality (Grant No: JCYJ20170817170110940).

\section{Disclosure}

The authors report no conflicts of interest in this work.

\section{References}

1. Munoz-Price L, Poirel L, Bonomo R, et al. Clinical epidemiology of the global expansion of Klebsiella pneumoniae carbapenemases. Lancet Infect Dis. 2013;13(9):785-796. doi:10.1016/S14733099(13)70190-7

2. Zhu J, Sun L, Ding B, et al. Outbreak of NDM-1-producing Klebsiella pneumoniae ST76 and ST37 isolates in neonates. Eur J Clin Microbiol Infect Dis. 2016;35(4):611-618. doi:10.1007/ s10096-016-2578-z

3. Chang-Ro L, Jung L, Kwang P, Young K, Byeong J, Sang L. Global dissemination of carbapenemase-producing Klebsiella pneumoniae: epidemiology, genetic context, treatment options, and detection methods. Front Microbiol. 2016;7:895. doi:10.3389/ fmicb.2016.00895

4. Eva H, Hasan E, Josefin S, et al. Resistance mechanisms and population structure of highly drug resistant Klebsiella in Pakistan during the introduction of the carbapenemase NDM-1. Sci Rep. 2019;9 (1):2392. doi:10.1038/s41598-019-38943-7

5. Zhou J, Li G, Ma X, Yang Q, Yi J. Outbreak of colonization by carbapenemase- producing Klebsiella pneumoniae in a neonatal intensive care unit: investigation, control measures and assessment. Am J Infect Control. 2015;43(10):1122-1124. doi:10.1016/j. ajic.2015.05.038

6. Hasan E, Nancy W, Jonathan J, et al. Phylogenetic analysis of Klebsiella pneumoniae from hospitalized children, Pakistan. Emerg Infect Dis. 2017;23(11):1872-1875. doi:10.3201/eid2311.170833

7. Patrice N, Thierry N, Poirel L. Global spread of carbapenemase producing Enterobacteriaceae. Emerg Infect Dis. 2011;17 (10):1791-1798. doi:10.3201/eid1710.110655

8. Poirel L, Hombrouk-Alet C, Freneaux C, Bernabeu S, Nordmann P. Global spread of New Delhi metallo- $\beta$-lactamase. Lancet Infect Dis. 2010;10(12):832. doi:10.1016/S1473-3099(10)70279-6

9. Cuzon G, Ouanich J, Gondret R, Naas T, Nordmann P. Outbreak of OXA-48-positive carbapenem-resistant Klebsiella pneumoniae isolates in France. Antimicrob Agents Chemother. 2011;55 (5):2420-2423. doi:10.1128/AAC.01452-10

10. Zhang X, Li X, Wang M, et al. Outbreak of NDM-1-producing Klebsiella pneumoniae causing neonatal infection in a teaching hospital in mainland China. Antimicrob Agents Chemother. 2015;59 (7):4349-4351. doi:10.1128/AAC.03868-14

11. Hu F, Guo Y, Zhu D, et al. Resistance trends among clinical isolates in China reported from CHINET surveillance of bacterial resistance, 2005-2014. Clin Microbiol Infect. 2016;22:S9-S14. doi:10.1016/j. cmi.2016.01.001

12. Findlay J, Hopkins L, Loy R, et al. OXA-48-like carbapenemases in the UK: an analysis of isolates and cases from 2007 to 2014 J Antimicrob Chemother. 2017;72(5):1340-1349. doi:10.1093/jac/ dkx012

13. Yin D, Dong D, Li K, et al. Clonal dissemination of OXA-232 carbapenemase-producing Klebsiella pneumoniae in neonates. Antimicrob Agents Chemother. 2017;61(8):e00385-17. doi:10.1128/ AAC.00385-17
14. Robert C. An overview of harms associated with $\beta$-lactam antimicrobials: where do the carbapenems fit in? Crit Care. 2008;12(S4):S3. doi:10.1186/cc6819

15. Haiyan L, Yu F, Ke M, Lu L, Alan M, Zhiyong Z. The co-transfer of plasmid-borne colistin-resistant genes mcr-1 and mcr-3.5, the carbapenemase gene blaNDM-5 and the $16 \mathrm{~S}$ methylase gene rmtB from Escherichia coli. Sci Rep. 2019;9(1):696. doi:10.1038/s41598-01837125-1

16. Kim Z, Angela H, Gerlinde P, Hester B, Albert N, Leo S. The Carbapenem Inactivation Method (CIM), a simple and low-cost alternative for the carba NP test to assess phenotypic carbapenemase activity in gram-negative rods. PLoS One. 2015;10(3):e0123690. doi:10.1371/journal.pone.0123690

17. Patel JB, Cockerill FR, Bradford PA. M100-S25 performance standards for antimicrobial susceptibility testing; Twenty-fifth informational supplement. 2019.

18. The European Committee on Antimicrobial Susceptibility Testing and Clinical and Laboratory Standards Institute. Recommendations for MIC determination of colistin (polymyxin E) as recommended by the joint CLSI-EUCAST Polymyxin Breakpoints Working Group. 2016.

19. Poirel L, Walsh T, Cuvillier V, Nordmann P. Multiplex PCR 344 for detection of acquired carbapenemase genes. Diagn Microbiol Infect. 2011;70(1):119-123. doi:10.1016/j.diagmicrobio.2010.12.002

20. Ejaz H, Ahmad M, Younas S, et al. Molecular epidemiology of extensively-drug resistant Acinetobacter baumannii sequence type 2 co-harboring bla $_{\mathrm{NDM}}$ and bla $\mathrm{OXX}_{\mathrm{OA}}$ from clinical origin. Infect Drug Resist. 2021;14:1931-1939. doi:10.2147/IDR.S310478

21. Naas T, Vandel L, Sougakoff W, Livermore D, Nordmann P. Cloning and sequence analysis of the gene for a carbapenem-hydrolyzing class A beta-lactamase, Sme-1, from Serratia marcescens S6. Antimicrob Agents Chemother. 1994;38(6):1262-1270. doi:10.1128/ AAC.38.6.1262

22. Dallenne C, Da A, Decre D, Favier C, Arlet G. Development of a set of multiplex PCR assays for the detection of genes encoding important $\beta$-lactamases in Enterobacteriaceae. J Antimicrob Chemother. 2010;65(3):490-495. doi:10.1093/jac/dkp498

23. Hui H, Haijian Z, Haishan L, et al. Optimization of pulse-field gel electrophoresis for subtyping of Klebsiella pneumoniae. Int J Environ Res Public Health. 2013;10(7):2720-2731. doi:10.3390/ ijerph 10072720

24. Andersson P, Tong YC, Bell JM, Turnidge JD, Giffard PM, Mokrousov I. Minim typing - a rapid and low cost MLST based typing tool for Klebsiella pneumoniae. PLoS One. 2012;7(3):e33530. doi:10.1371/journal.pone.0033530

25. Carattoli A, Bertini A, Villa L, Falbo V, Hopkins L, Threlfall J. Identification of plasmids by PCR-based replicon typing. $J$ Microbiol Methods. 2005;63(3):219-228. doi:10.1016/j. mimet.2005.03.018

26. Almogbel M, Altheban A, Alenezi M, et al. CTX-M-15 positive Escherichia coli and Klebsiella pneumoniae outbreak in the neonatal intensive care unit of a maternity hospital in Ha'il, Saudi Arabia. Infect Drug Resist. 2021;14:2843-2849. doi:10.2147/IDR.S317079

27. Berberian G, Brizuela M, Rosanova T, et al. Multidrug resistant gram-negative infections in neonatology. Arch Argent Pediatr. 2019;117(1):6-11. doi:10.5546/aap.2019.eng.6

28. Hasan E, Badr A, Mutaz H, et al. Molecular analysis of the antibiotic resistant NDM-1 gene in clinical isolates of Enterobacteriaceae. Clin Lab. 2020;60:XXX. doi:10.7754/Clin.Lab.2019.190727

29. Hasan E, Sonia Y, Muhammad Q, et al. Molecular epidemiology of extensively drug-resistant $\mathrm{mcr}$ encoded colistin-resistant bacterial strains co-expressing multifarious $\beta$-lactamases. Antibiotic. 2021;10 (4):46. doi:10.3390/antibiotics.10040467

30. Kelly L, Kathryn E. Klebsiella pneumoniae as a key trafficker of drug resistance genes from environmental to clinically important bacteria. Curr Opin Microbiol. 2018;45:131-139. doi:10.1016/j.mib.2018.04.004 
31. Monika D, Martina M, Hana D, et al. High prevalence of Salmonella and IMP-4-producing Enterobacteriaceae in the silver gull on Five Islands, Australia. J Antimicrob Chemother. 2016;71(1):63-70. doi: $10.1093 / \mathrm{jac} / \mathrm{dkv} 306$

32. Cailin L, Shangshang Q, Hui X, et al. New Delhi Metallo- $\beta$ Lactamase 1(NDM-1), the dominant carbapenemase detected in carbapenem-resistant Enterobacter cloacae from Henan Province, China. PLoS One. 2015;10(8):e0135044. doi:10.1371/journal. pone.0135044

33. Liu Y, Wan L, Deng Q, Cao X, Yu Y, Xu F. First description of NDM-1-, KPC-2-, VIM-2- and IMP-4-producing Klebsiella pneumoniae strains in a single Chinese teaching hospital. Epidemiol Infect. 2015;143(2):376-384. doi:10.1017/S0950268814000995

34. Jinrong Y, Shuli P, Xiaojiong J, et al. Multidrug resistance mechanisms of carbapenem resistant Klebsiella pneumoniae strains isolated in Chongqing, China. Ann Lab Med. 2017;37(5):398-407. doi:10.3343/alm.2017.37.5.398

35. Shudan C, Fupin H, Xiaogang X, et al. High prevalence of KPC2-type carbapenemase coupled with CTX-M-type extended-spectrum $\beta$-lactamases in carbapenem-resistant Klebsiella pneumoniae in a teaching hospital in China. Antimicrob Agents Chemother. 2011;55(5):2493-2494. doi:10.1128/AAC.00047-11

36. Bonura C, Giuffre M, Aleo A, et al. An update of the evolving epidemic of blaKPC carrying Klebsiella pneumoniae in Sicily, Italy, 2014: emergence of multiple non-ST258 clones. PLoS One. 2015;10 (7):e0132936. doi:10.1186/cc6819
37. Yoon E, Yang J, Kim J, et al. Carbapenemase-producing Enterobacteriaceae in South Korea: a report from the National Laboratory Surveillance System. Future Microbiol. 2018;13:771-783. doi:10.2217/fmb-2018-0022

38. Castanheira M, Farrell SE, Wanger A, et al. Rapid expansion of KPC-2- producing Klebsiella pneumoniae isolates in two Texas hospitals due to clonal spread of ST258 and ST307 lineages. Microb Drug Resist. 2013;19(4):295-297. doi:10.1089/mdr.2012.0238

39. Paola B, Elvira G, Magaly P, et al. The successful containment of a hospital outbreak caused by NDM-1-producing Klebsiella pneumoniae ST307 using active surveillance. PLoS One. 2019;14(2): e0209609. doi:10.1371/journal.pone.0209609

40. Lu L, Yu F, Guangmin T, et al. Carbapenem-resistant isolates of the Klebsiella pneumoniae complex in Western China: the common ST11 and the surprising hospital-specific types. Clin Infect Dis. 2018;67 (S2):S263-S265. doi:10.1093/cid/ciy662

41. Dongxing T, Fen P, Chun W, Yan S, Hong Z. Resistance phenotype and clinical molecular epidemiology of carbapenem-resistant Klebsiella pneumoniae among pediatric patients in Shanghai. Infect Drug Resist. 2018;11:1935-1943. doi:10.2147/IDR.S175584

42. Rémy B, Agnès J, Adriana C, et al. Emergence of new non-clonal group 258 high-risk clones among Klebsiella pneumoniae carbapenemase-producing K. pneumoniae isolates, France. Emerg Infect Dis. 2021;26(6):1212-1220. doi:10.3201/eid2606.191517
Infection and Drug Resistance

\section{Publish your work in this journal}

Infection and Drug Resistance is an international, peer-reviewed openaccess journal that focuses on the optimal treatment of infection (bacterial, fungal and viral) and the development and institution of preventive strategies to minimize the development and spread of resistance. The journal is specifically concerned with the epidemiology of

\section{Dovepress}

antibiotic resistance and the mechanisms of resistance development and diffusion in both hospitals and the community. The manuscript management system is completely online and includes a very quick and fair peerreview system, which is all easy to use. Visit http://www.dovepress.com/ testimonials.php to read real quotes from published authors. 